\title{
QUANTITATIVE SCALAR DISSIPATION RATE MEASUREMENTS IN VORTEX-PERTURBED COUNTERFLOW DIFFUSION FLAMES
}

\author{
DIMITRIOS C. KYRITSIS, VITO S. SANTORO AND ALESSANDRO GOMEZ \\ Yale Center for Combustion Studies, Department of Mechanical Engineering \\ Yale University \\ 9 Hillhouse Avenue \\ New Haven, CT 06520, USA
}

\begin{abstract}
Even though the scalar dissipation rate at the stoichiometric surface, $\chi_{\text {stoich }}$, is recognized to be the most fundamental fluid time scale in laminar diffusion flames, their structure and extinction behavior are often characterized simply in terms of strain rate, a much more easily measurable observable. Yet, the two variables are different, especially in unsteady flamelets. An experimental technique based on line Raman imaging of major species is presented for the quantitative measurement of $\chi_{\text {stoich }}$ in vortex-perturbed counterflow diffusion flames. Three formulations are evaluated, and it is shown that a formulation based on $\mathrm{N}_{2}$-mass fraction is the most appropriate, provided that $\mathrm{N}_{2}$ is experimentally accessible and that there is no significant preferential diffusion. The technique is used to compare vortex-perturbed and quasi-steady extinction. The thesis that for a given composition of the counterflowing streams, extinction occurs at a given value of $\chi_{\text {stoich }}$, irrespective of the mode of perturbation, steady or unsteady, is verified experimentally and is contrasted with the observation that vortex-perturbed flames can sustain an almost double strain rate at extinction compared to steadily strained ones. The effect of two-dimensional phenomena on the results is discussed. Finally, a promising approximation of $\chi_{\text {stoich }}$ using estimates of the thickness of mixing layer from temperature profiles, with significant simplifications in the required measurements, is investigated.
\end{abstract}

\section{Introduction}

The interaction of laminar diffusion flames and vortices is a problem of both practical and theoretical interest. The phenomenon can be observed in numerous flame configurations, and its analysis is the basis of flamelet models for turbulent combustion. Most of the recent theoretical, computational, and experimental work on the subject is reviewed in Ref. [1]. As for the case of steady laminar diffusion flames, also for these manifestly unsteady flamelets, the most relevant parameter to characterize their structure and their behavior near extinction is a suitably defined Damköhler number that is the ratio of a mechanical time and a chemical time. As a mechanical time, it is often convenient to use an inverse strain rate, which leads to the definition of Damköhler's first similarity group. But, it is generally accepted that a better suited mechanical time is a diffusive time at the stoichiometric surface where most of the chemistry sustaining the flame is, which yields Damköhler's second similarity group [2]. This diffusion time is the inverse of the scalar dissipation rate at the stoichiometric surface, which, in turn, is defined as $\chi_{\text {stoich }}=2 D(\nabla Z)_{\text {stoich }}^{2}$, where $D$ is a characteristic gaseous thermal diffusivity and $Z$ is the mixture fraction, that is, the fraction of the local mixture that originated in the fuel stream [3]. It can be shown [4] that the rate of fuel depletion is proportional to $\chi_{\text {stoich }}$, a property which strain rate clearly lacks. The maximum value of $\chi_{\text {stoich }}$ corresponds to the maximum flow rate of fuel that can be consumed by the flame and occurs at extinction. The distinction between strain rate and scalar dissipation rate is not critical in steady flamelets, since the two variables are proportional to each other. Quite different is the case of flames subject to time-dependent perturbations, such as vortices, in which the scalar dissipation rate depends on the time history of the strain rate. Yet even for such flames, including those undergoing interaction with a vortex, the intuitive, and therefore most common, approach has been to study the phenomenon in terms of the velocity field and use the strain rate imposed on the flame as the controlling parameter [5-7]. An example of the significant difference between the two variables is offered by previous measurements showing that extinction in vortex perturbed counterflow diffusion flames occurs at roughly double the strain rate compared to steadily strained ones, whereas theory suggests that the two flames should extinguish at the same scalar dissipation rate, since the inner reactive-diffusive layer behaves quasi-steadily [8,9]. Refs. [10] and [11], in which the effect of curvature on flame extinction is studied from both scalar dissipation rate and strain 
rate perspectives, provide yet another example of the discrepancy between the two variables.

Our objectives are manifold. First, we provide an experimental technique for the quantitative measurement of $\chi_{\text {stoich }}$ and use it to estimate this parameter for quasi-steady and vortex-perturbed flames approaching extinction. As far as diagnostics is concerned, the study of flame-vortex interaction has yielded two-dimensional images of various observables in the toroidal mixing layers $[6,12]$. A technique based on Raman spectroscopy, very similar to the one to be discussed here, was used in Ref. [7] for number density measurements; however, the potential of acquiring a measurement of the mixture fraction $(Z)$ from the number density information was not evaluated.

Second, once a reliable measurement technique of $\chi_{\text {stoich }}$ is available, its application in vortex-induced and quasi-steady extinction is pursued, as a followup to the work in Refs. $[8,9]$, to test experimentally the hypothesis that $\chi_{\text {stoich }}$ at extinction is independent of the mode of perturbation. Once this hypothesis is experimentally confirmed, it can be investigated in terms of scalar dissipation rate whether the curvature effects on extinction reported for $\mathrm{H}_{2}$ diffusion flames in Ref. [11] persist in flames of Lewis number close to unity. If the phenomenon is studied only in terms of strain rate, unsteadiness and curvature are, in fact, interlaced, and the effect of each cannot be isolated.

Third, regardless of the particular application, in view of the significant experimental resources and effort a direct measurement of $\chi_{\text {stoich }}$ requires, we identify easily measurable observables which could approximate $\chi_{\text {stoich }}$ to a reasonable degree. More specifically, since $\chi_{\text {stoich }}$ is expected to correlate with the thickness of the mixing layer [13], that can be represented by the thickness of the temperature profile, we verify experimentally if such a shortcut is promising.

\section{Experimental Apparatus}

The burner used for the current study has been described in detail in Refs. [8,9]. A horizontal, gaseous counterflow diffusion flame was established between two vertical nozzles with oxygen and nitrogen diluent being fed from the upper side and methanol fuel and nitrogen from the lower side. The diameter of both nozzles was $12.7 \mathrm{~mm}$, and the distance between them was $13.5 \mathrm{~mm}$. The fuel mass fraction at the fuel nozzle was constant $Y_{\mathrm{CH} 3 \mathrm{OH}}=0.18$, whereas the oxidizer mass fraction $Y_{\mathrm{O} 2}$ at the oxidizer nozzle varied from 0.55 to 1 . The average speed at the oxidizer nozzle varied from 0.63 to $0.80 \mathrm{~m} / \mathrm{s}$ and at the fuel nozzle from 0.62 to $0.90 \mathrm{~m} / \mathrm{s}$ for the flames studied here. The flame was perturbed at a frequency of $10 \mathrm{~Hz}$ by laminar vortices according to the scheme described in Refs. $[8,9]$. For the bulk of the results reported here, a suitably designed voltage function was applied to a loudspeaker causing the latter to force fluid impulsively through a $1.5 \mathrm{~mm}$ tube positioned in the oxidizer stream. The presence of two-dimensional effects on the results was also investigated during experiments with $0.5-3.0 \mathrm{~mm}$ diameter tubes. The vortices start interacting with the flame 8-10 ms after their actuation and cause extinction in the center of the circular horizontal flame 12-14 ms after the actuation. The flame reignites at approximately $35-40 \mathrm{~ms}$ and resumes its flat shape long before the completion of the $100 \mathrm{~ms}$ period of the phenomenon.

A Nd:YAG laser delivering $100 \mathrm{~mJ} /$ pulse at $532 \mathrm{~nm}$ was used as an excitation source. The laser beam was focused to an approximately $200 \mu \mathrm{m}$ thick and $30 \mathrm{~mm}$ long horizontal line with the use of a $750 \mathrm{~mm}$ planoconvex lens. Scans were taken in the direction perpendicular to the flame $(y)$. The horizontal line was imaged vertically at the entrance slit of an $300 \mathrm{~mm}$ spectrograph with a $50 \mathrm{~mm}$, f1.8 lens. To reject stray light from the burner hardware, an OG 550, $3 \mathrm{~mm}$ thick glass filter was used. Dispersion was achieved with a $68 \times 68 \mathrm{~mm}$ ruled grating with 1200 grooves $/ \mathrm{mm}$ and a $500 \mathrm{~nm}$ blaze wavelength. The spectrograph output was imaged on the photocathode of a image intensifier. The intensifier was operated at a full luminous gain of 10,000 (nominal) and gated for $300 \mathrm{~ns}$, and its phosphor was coupled optically with two $50 \mathrm{~mm}$, f1.8 lenses to an Apogee AP7 camera. Depending on the particular spatial location with respect to the flame and the detected species, it was necessary to integrate on the camera chip for 50-200 s (500-2000 laser shots) to achieve an acceptable $\mathrm{S} / \mathrm{N}$.

Raman signal from a $3 \mathrm{~mm}$ segment of the spectral lines around the centerline was integrated to account for the noise in the intensified camera output. The mild effect of temperature on Raman cross section, mainly for $\mathrm{CO}_{2}$ and $\mathrm{H}_{2} \mathrm{O}$, was accounted for introducing the corrections suggested in Ref. [14]. For $\mathrm{N}_{2}, \mathrm{O}_{2}$, and $\mathrm{CH}_{3} \mathrm{OH}$, the number densities at the nozzle exits were calculated from the measured flow rates in the feed streams, and the corresponding calibration constants were determined from the recorded signal at the location closest to the appropriate nozzle. For the combustion products $\left(\mathrm{CO}_{2}\right.$, $\mathrm{CO}$, and $\mathrm{H}_{2} \mathrm{O}$ ), the calibration constants were calculated by correcting the constant used for nitrogen for Raman cross-section of the relevant species and photocathode sensitivity at the relevant wavelengths. The process of calibrating the Raman signal and the determination of the necessary correction constants are described in detail in Refs. $[14,15]$.

\section{Results and Discussion}

To calculate mass fractions from the measured number densities, it was assumed that the mixture 


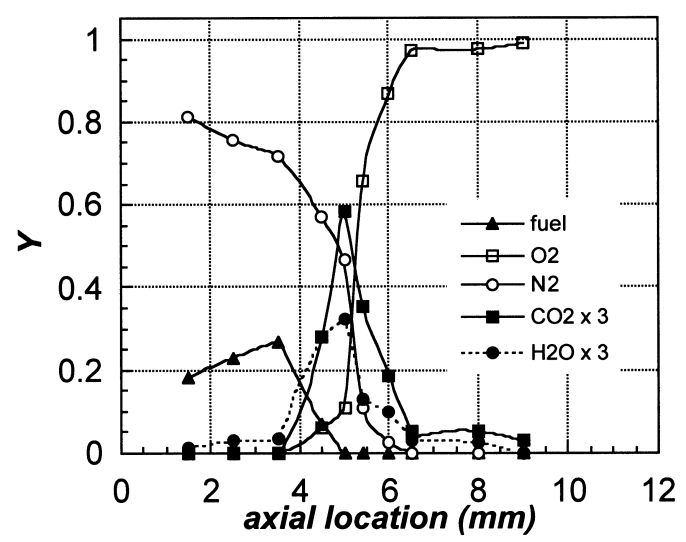

FIG. 1. Measured mass fraction profiles of major species.

consisted only of the six measured species. Results of the mass fraction measurement for a flame with $Y_{\mathrm{CH} 3 \mathrm{OH}}=0.18$ and $Y_{\mathrm{N} 2}=0.82$ at the fuel nozzle and $Y_{\mathrm{O} 2}=1$ at the oxidizer nozzle are shown in Fig. 1 at time $t=10 \mathrm{~ms}$ after vortex actuation. At this time, the vortex has not interacted with the flame yet. Comparison with computed results for the same flame is provided in Ref. [15] and shows good agreement. The measurement of $\mathrm{CO}$ number density in the results presented here is of marginal quality and is taken with a $\mathrm{S} / \mathrm{N}$ of the order of 1 . This is due to the weakness of CO Raman, which is difficult to discriminate against a strong cross-talk from the adjacent $\mathrm{N}_{2}$ signal. The resulting $Y_{\mathrm{CO}}$ always remains below $2 \%$. Since $Y_{\mathrm{CO}}$ is underestimated in the measurement, the mass fraction of fuel appears artificially increased at the boundary of the fuel depletion region, where the maximum values of $Y_{\mathrm{CO}}$ are expected. A more accurate measurement of $\mathrm{CO}$ would require significantly longer on-chip integrations, which would prolong the experimental effort without altering the conclusions of this work. It should also be kept in mind that the Raman line of methanol used here has lower cross-section than methane Raman, which is used as a fuel in similar studies [7]. The related error is therefore higher and could account for part of the fuel overshoot observed in Fig. 1.

It can be seen from Fig. 1 that the thickness of the mixing layer is approximately $2 \mathrm{~mm}$. The thickness of this layer decreases as the vortex interacts with the flame, and the strain rate and $\chi_{\text {stoich }}$ increase, until extinction occurs. Occurrence of extinction can be determined from the Raman results when no combustion products are detected along the centerline.

From the measured mass fractions, it is possible to obtain the mixture fraction profile necessary for the measurement of $\chi_{\text {stoich }}$. The mixture fraction is defined as $Z=\left(\beta-\beta_{\mathrm{ox}}\right) /\left(\beta_{\mathrm{fu}}-\beta_{\mathrm{ox}}\right)$, where fu and ox denote fuel and oxidizer nozzle, respectively, and $\beta$ is a conserved scalar. $Z$ and $\beta$ have been measured extensively with single-shot, two-dimensional Raman and Rayleigh imaging in turbulent flows [1620]. The only factor determining the choice of the particular formulation of $\beta$ for two-dimensional, single-shot measurements is $\mathrm{S} / \mathrm{N}$. This need not be the case for measurements in a repeatable, laminar configuration. Interestingly, the assumptions associated with each of the formulations of $\beta$ and the accuracy of corresponding measurements are discussed in detail in Ref. [21], but only theoretically, without data from experiments on specific configurations. By definition of the conserved scalar, the best experimental observable for it would be the mass fraction of an inert diluent with molecular weight close to the average molecular weight of the mixture. The main problem with this approach is that inert diluents are practically diagnostically inaccessible. Fielding et al. [17] investigate the possibility of using $\mathrm{N}_{2}$ mass fraction to perform two-dimensional measurements of $\mathrm{Z}$, but the two-dimensional Raman signal of $\mathrm{N}_{2}$ was too weak to use this formulation in turbulent environments. However, $\mathrm{N}_{2}$ line Raman signal can be measured with $\mathrm{S} / \mathrm{N}$ higher than the one reported in Refs. [17,22] for periodic phenomena, by phase locking on the periodic perturbation and integrating on the detector.

In principle, with the mass fraction information available here, $\beta$ could be formulated in three different ways: $\beta=Y_{\mathrm{C}}, \beta=Y_{\mathrm{CH} 3 \mathrm{OH}}-Y_{\mathrm{O} 2} / s$, and $\beta=Y_{\mathrm{N} 2} . Y$ is the mass fraction, $s$ is the stoichiometric oxygen-to-methanol mass ratio, and the subscript $\mathrm{c}$ refers to carbon atom. The first formulation is the most fundamental since it ensues from atom conservation. However, for the calculation of $Y_{\mathrm{c}}$, an accurate knowledge of $Y_{\mathrm{CO}}$ is necessary, which is problematic, because of the weakness of CO Raman. The second formulation implies one-step kinetics and is expected to be problematic in the region of the flame where fuel pyrolysis takes place. This formulation is also affected the most by the overshoot in the fuel mass fraction measurement described in the previous paragraph. The last formulation implies that $\mathrm{N}_{2}$ is inert, which is a reasonable assumption.

In Fig. 2, the mixture fraction is reported as a function of axial location calculated with each of the above formulations for the conditions of Fig. 1. When the carbon atom or the fuel-oxidizer formulations were used, abrupt changes in $|\nabla Z|$ or even non-monotonic behavior of $Z$ was observed. The disadvantages of these two formulations are independent of the particular combustion configuration and suggest that a $\mathrm{N}_{2}$-based formulation is preferable. Compared to the more complicated formulations of $Z$ discussed in Ref. [21], a $\mathrm{N}_{2}$-based one has the advantage that it requires the simultaneous measurement of less primary quantities, thus making the measurement both faster and less vulnerable to error 


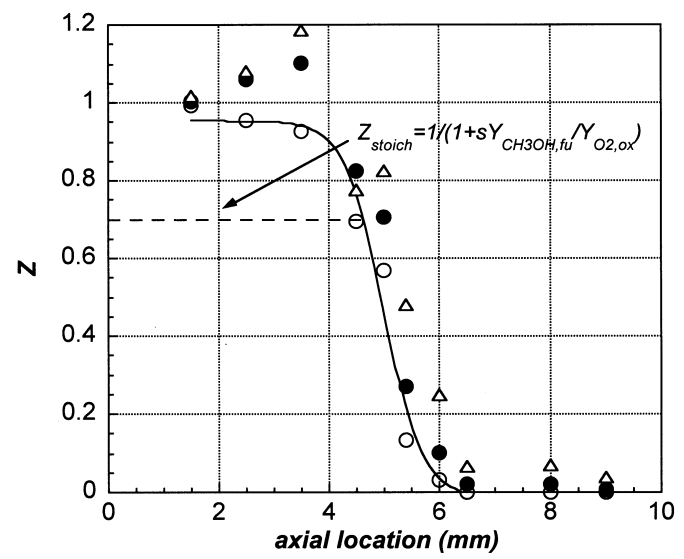

FIG. 2. Measured mixture fraction profiles using the fuel-oxidizer pair $(\bullet)$, carbon atom $(\triangle)$, and nitrogen molecule $(\hat{x})$ formulations. The solid line indicates the complementary error function behavior predicted by a thermal diffusive model with one-step kinetics [13].

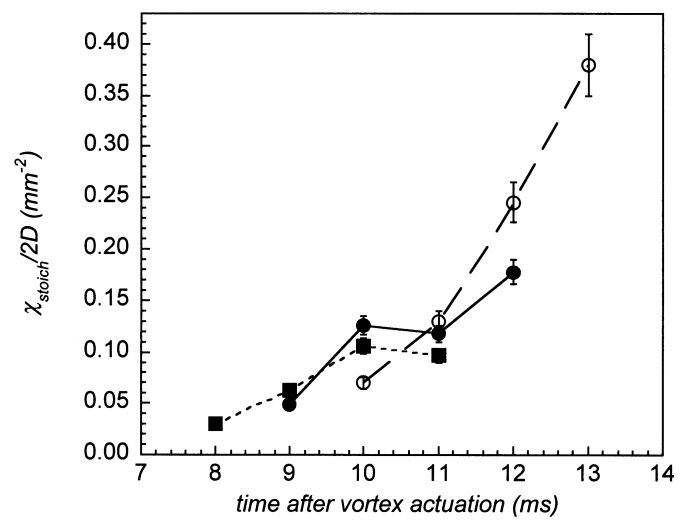

FIG. 3. Temporal evolution of the scalar dissipation rate during the last $4 \mathrm{~ms}$ before extinction for flames with $Y_{\mathrm{CH} 3 \mathrm{OH}}=0.18$ and $Y_{\mathrm{O} 2}=0.55(\mathbf{\square}), Y_{\mathrm{O} 2}=0.75(\bullet)$, and $Y_{\mathrm{O} 2}=1.00(\hat{\mathrm{x}})$.

propagation. The phenomena of preferential diffusion, that can be accommodated by some of these definitions, are not a significant concern in flames in which fuel, oxidizer, and diluent have approximately equal molecular weights. We conclude that, in general, in configurations where $\mathrm{N}_{2}$ Raman is accessible with an acceptable $\mathrm{S} / \mathrm{N}$, a $\mathrm{N}_{2}$-based formulation should be preferred for the measurement of $Z$. Unfortunately, this is not the case for two-dimensional, instantaneous measurements in turbulent flows [17]. The technique described here can be applied even when preferential diffusion is present. In such a case, caution should be used for the proper definition of the mixture fraction.
The location of the stoichiometric surface can be determined from the data of Fig. 2 taking into account that $Z_{\text {stoich }}=1 /\left(1+s Y_{\mathrm{CH} 3 \mathrm{OH}, \mathrm{fu}} / Y_{\mathrm{O} 2, \mathrm{Ox}}\right)$. To calculate the scalar dissipation rate for a specified effective diffusivity, $\left|\nabla Z_{\text {stoich }}\right|$ needs to be evaluated. Theoretical analysis $[8,9]$ suggesting that $Z$ scales as the $\operatorname{erfc}(\bar{y})$, where $\bar{y}$ is a non-dimensional distance from the flame sheet and erfc is the complementary error function can guide any smoothing of the inevitable experimental noise. We can thus curve fit a function of the form $Z=A \cdot \operatorname{erfc}(\bar{y})=A \cdot \operatorname{erfc}((y-B) / C)$ to the measured distribution of $Z$ and calculate its derivative at $Z=Z_{\text {stoich }}$. Typically, regression coefficients are $R>$ 0.98 . To study flame extinction, the procedure was repeated with an increment in time of $1 \mathrm{~ms}$ for the last $4 \mathrm{~ms}$ before extinction. The last value of $\left|\nabla Z_{\text {stoich }}\right|$ is a measurement of $\left|\nabla Z_{\text {stoich }}\right|$ at extinction.

Results are reported in Fig. 3, where scalar dissipation rate is presented as a function of time for three flames of varying oxidizer stream composition. The volumetric flow rates of the feed streams were kept constant and $Y_{\mathrm{CH} 3 \mathrm{OH}}=0.18$ at the fuel nozzle. The extinction point is the rightmost data point of each data set, at which an increase of $\chi_{\text {stoich }}$ by a factor of 4-8 is observed with respect to its value calculated $4 \mathrm{~ms}$ before extinction, when we can assume that the flame has not been perturbed by the vortex yet. The error in the measurement of $\left|\nabla Z_{\text {stoich }}\right|$ $=|d Z / d y|_{\text {stoich }}$ was estimated as follows. The error in the raw Raman signal was approximated by the standard deviation of the recorded signal within the window of integration. The propagation of the error from each raw signal allowed for the estimate of the error associated with Z. An estimate of the error $\left|\nabla Z_{\text {stoich }}\right|$ was achieved from the error in the two measured points neighboring $Z_{\text {stoich }}$ by approximating the spatial derivative as

$$
\left(\frac{d Z}{d y}\right) \approx \frac{Z(y+\Delta y)-Z(y-\Delta y)}{2 \Delta y}
$$

where $\Delta y$ is the spatial increment of the measurements. The maximum error in the measurement of species number density is on the order of $10 \%$. From $\left|\nabla Z_{\text {stoich }}\right|, \chi_{\text {stoich }}$ can be calculated, using well-established approximations for the mixture diffusivity as a function of temperature. Error propagation can be used to estimate a maximum error of $15 \%$ in the $\chi_{\text {stoich }}$ measurement. Indicative error bars are shown in Fig. 3.

In Fig. 4 , the ratio of $\chi_{\text {stoich }}$ at extinction for vortexinduced perturbation over the corresponding value of $\chi_{\text {stoich }}$ for quasi-steady extinction is presented as a function of $Y_{\mathrm{O} 2}$ in the oxidizer stream. Quasi-steady extinction was achieved by gradually increasing the flow rates of the counterflowing streams until extinction is achieved. Notice that, if we assume that the temperature at extinction is independent of the mode of excitation, 


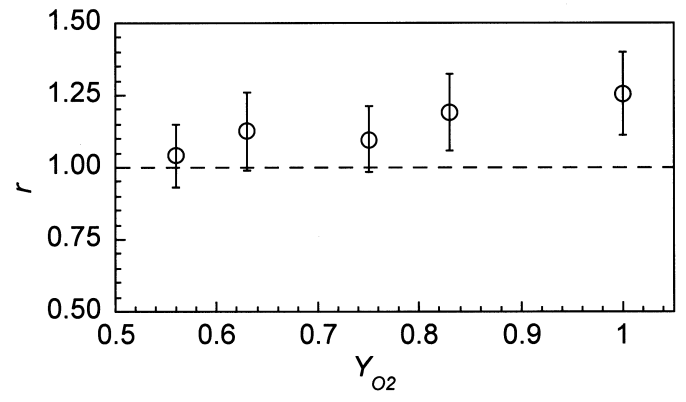

FIG. 4. The ratio of scalar dissipation rate at extinction for vortex-induced extinction over the corresponding value for quasi-steady extinction $(r)$ for flames of varying composition of the oxidizer stream.

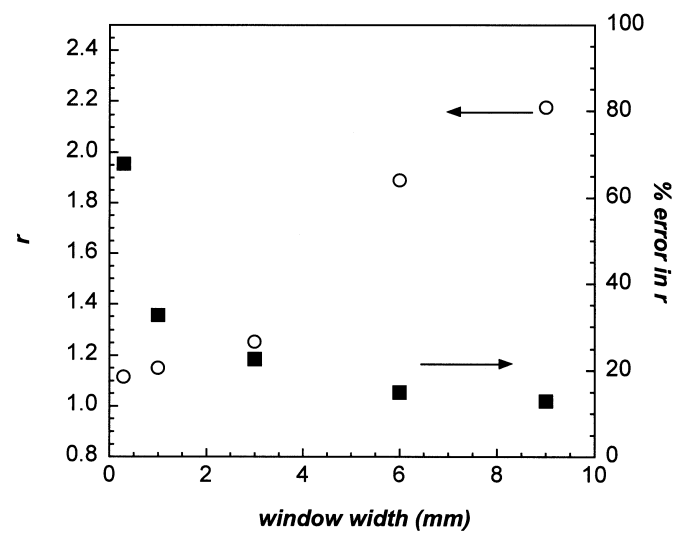

FIG. 5. The ratio $r(\hat{\mathrm{x}})$ and the relative error in its measurement ( $\mathbf{\square}$ ) as a function of the width of integration of the raw Raman signal for $Y_{\mathrm{O} 2}=1.00$.

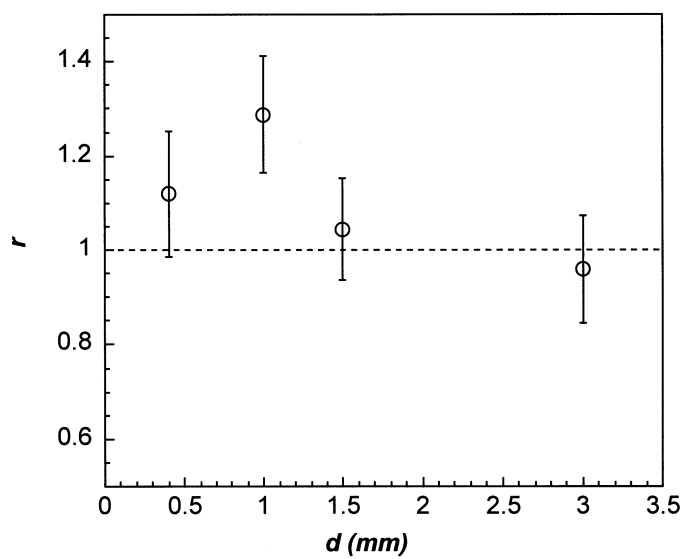

FIG. 6. The effect of the injecting tube diameter $(d)$ on the ratio of scalar dissipation rate at extinction for vortexinduced extinction over the corresponding value for quasisteady extinction for $Y_{\mathrm{O} 2}=0.55$.

$$
\begin{aligned}
r= & \frac{\chi_{\text {stoich,ext,vort }}}{\chi_{\text {stoich,ext,qs }}}=\frac{2 D_{\text {ext,vort }}(\nabla Z)_{\text {stoich,ext,vort }}^{2}}{2 D_{\text {ext,qs }}(\nabla Z)_{\text {stoich,ext,qs }}^{2}} \\
& \approx \frac{(\nabla Z)_{\text {stoich,ext,vort }}^{2}}{(\nabla Z)_{\text {stoich,ext,qs }}^{2}}
\end{aligned}
$$

The error bars on the ratio $r$ are calculated from the error on $\left|\nabla Z_{\text {stoich }}\right|$ using equation 2. The maximum error is $25 \%$. The proximity of $r$ to unity should be compared with the results of Refs. [8,9], which show that the corresponding ratio in terms of strain rate in the same flames is more than a factor of 2 . It is clear that $\chi_{\text {stoich }}$ is the fundamental parameter controlling flame extinction, relatively invariant to the mode by which extinction is brought about.

Figure 4 shows that for most of the flames the distance of each separate value of $r$ from unity is within the experimental error of the measurement. However, the fact that for all studied flames, $r>1$ is worth further investigation. As explained previously, to account for the noise in the camera output, an integration of the Raman signal in a region $3 \mathrm{~mm}$ thick around the centerline was necessary. Since, for the vortex-perturbed flames, the flame sheet was slightly curved, this integration leads to an underestimation of the number density of the combustion products. As a result of this, the thickness of the mixing layer is artificially decreased and the scalar dissipation rate increased. This is not the case for a flat flame, for which integration can be performed along a line parallel to the flame without introducing systematic error. In Fig. 5, two different sources of error of the measurement are presented as a function of the width of the window of integration for a flame with $Y_{\mathrm{O} 2}=1$. The value of $r$ departs gradually from unity as the width of this window increases. Also in Fig. 5, the relative error on $r$ is presented, and it can be seen that it increases for narrowing integration windows, due to the increased error in the raw Raman signal. The final choice of a $3 \mathrm{~mm}$ wide window is a reasonable compromise between these two trends.

The presence of two-dimensional effects in the results reported here was investigated using vortices that emerged from tubes of varying diameter. In Fig. 6, $r$ is shown as a function of tube diameter for a flame with $Y_{\mathrm{O} 2}=0.55$, that is, the most diluted flame of the ones reported in Fig. 3. The particular flame was chosen to ensure that extinction could be achieved even with small size vortices. Within the experimental error of the technique employed here, no dependence of the value of $r$ at extinction on the diameter of the injecting tube can be detected. This result does not necessarily contradict already published findings on the effect of curvature on flame extinction $[10,11,23,24]$. The flames under consideration here lie on the fuel side of the stagnation plane, so the vortices emerging from the oxidizer side undergo a significant radial stretch before they 


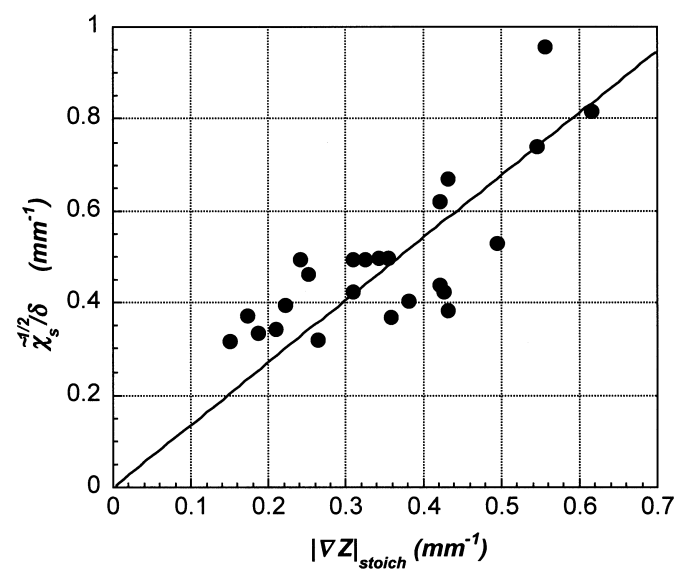

FIG. 7. Correlation of the temperature-based thickness $\delta$ of the mixing layer with $\left|\nabla Z_{\text {stoich }}\right|$.

interact with the flame. Flow visualization with alumina particles showed radii of curvature varying between 3 and $7 \mathrm{~mm}$, which are always larger than the mixing layer thickness by a factor on the order of 10 . Therefore, at best modest two-dimensional effects on $\chi_{\text {stoich }}[10]$ could be detected with the current configuration. The negligibility of these effects is compounded by the near-unity Lewis number of the present flames. The effect probably must be studied with a more complicated vortex delivery scheme that can deliver vortices of size comparable to the mixing layer thickness. Further comparisons with previous experiments are difficult, even for similar radii of curvature [11], because their results have been compiled in terms of strain rate. However, it is reminded that the strain rate at extinction is a function not only of curvature, but also of the time history of the particular perturbation $[8,9]$. Given that $\chi_{\text {stoich }}$ at extinction is independent of unsteady phenomena, curvature effects can be studied independently when analyzed in terms of scalar dissipation rate. In phenomenological terms, it should be noted that the line scans across the flame do not show the annular extinction phenomena suggested for $\mathrm{H}_{2}$ flames in Ref. [24].

Much as they provide a key variable describing the physics of diffusion flame extinction, direct measurements of $\chi_{\text {stoich }}$ require a significant amount of experimental effort and resources. A simplification of the experimental process could ensue from recognizing that $\left|\nabla Z_{\text {stoich }}\right|=\bar{\chi}_{\mathrm{s}}^{1 / 2} / \delta$, where $\delta$ is the mixing layer thickness and $\bar{\chi}_{s}^{1 / 2}$ is a non-dimensional constant, which is only a function of $Z_{\text {stoich }}$ [13]. The value of this constant only varies between 0.29 and 0.36 , in which case $\left|\nabla Z_{\text {stoich }}\right|$ is approximately inversely proportional to the thickness of the mixing layer. A way to establish a consistent definition of $\delta$ experimentally is to consider the temperature profile across this layer. Temperature can be derived from the Raman data as $T=P / n R$, where $n$ is the total number density. To minimize any arbitrariness due to experimental noise, a Gaussian curve is fitted to the data, since, theoretically, a Gaussian profile is to be expected $[8,9,13]$. The thickness of the mixing layer $\delta$ is defined as the standard deviation of the Gaussian. In Fig. $7, \bar{\chi}_{\mathrm{s}}^{1 / 2} / \delta$ is presented as a function of $\left|\nabla Z_{\text {stoich }}\right|$. The two quantities correlate linearly with a regression coefficient of 0.80 . This correlation must be investigated further, for example, in an experiment in which the $\mathrm{CO}$ measurement is of better quality or with measurements with a higher $\mathrm{S} / \mathrm{N}$, since it may imply that the tedious measurement of $\chi_{\text {stoich }}$ could be substituted by a relatively simple temperature measurement. In laminar environments, such measurements can be achieved either by a simple thermocouple scan or via thin-wire pyrometry for periodically perturbed flames. In turbulent environments, two-dimensional distributions of temperature have been acquired by fluorescence measurements of non-reacting dopants $[25,26]$. The relatively large thickness of the temperature layer and the high uncertainty implied by Fig. 7 suggest that application of this simpler technique in turbulent flames may be problematic. However, fast and reasonable estimates of $\chi_{\text {stoich }}$ can be achieved in laminar flames with minimal experimental resources.

\section{Summary and Conclusions}

Quantitative scalar dissipation rate measurements using line Raman imaging of major species are reported in vortex-perturbed and steadily strained diffusion flames. The goal was to provide a reliable tool for the measurement of the controlling parameter of such flames, which are usually studied in terms of strain rate. The most appropriate formulation of the mixture fraction for such measurements in laminar, periodic flows is one based on the mass fraction of the inert $\left(\mathrm{N}_{2}\right)$, because the normally weak $\mathrm{N}_{2}$ Raman can be detected with good signal-to-noise ratios with phase-locked measurements. The maximum error in the measurement of scalar dissipation rate was $15 \%$. The technique was used to compare vortex-perturbed and quasi-steady extinction. The measured scalar dissipation rate at extinction for the vortexperturbed flames was only between 5 and $26 \%$ higher than the one for quasi-steady extinction, compared to previous measurements in the same flames for which the corresponding difference in terms of strain rate were of the order of a factor of 2 . As a result, theoretical expectations that the scalar dissipation rate at the stoichiometric surface should be the controlling parameter of the phenomenon are confirmed. The $5 \%$ to $26 \%$ discrepancy is currently attributed to a systematic error associated with the 
integration in a window around the centerline, which is necessary for the acquisition of Raman signal with an acceptable $\mathrm{S} / \mathrm{N}$. For the vortices investigated here, two-dimensional (curvature) effects were not important. A correlation was found between scalar dissipation rate and the thickness of the temperature profile across the flame, which may offer an easy experimental alternative to the tedious scalar dissipation rate measurements based on Raman spectroscopy.

\section{Acknowledgments}

The support of NSF, grant CTS-9904296 (Dr. F. Fischer, Contract Monitor) is gratefully acknowledged. The authors would like to acknowledge Prof. M. B. Long of Yale University for his insightful suggestions on the implementation of the Raman diagnostic technique.

\section{REFERENCES}

1. Renard, P. H., Thévenin, D., Rolon, J. C., and Candel, S., Prog. Energy Combust. Sci. 26:225-282 (2000).

2. Williams, F. A., Prog. Energy Combust. Sci. 26:657682 (2000).

3. Bilger, R. W., Combust. Sci. Technol. 13:155-170 (1976).

4. Williams, F. A., Combustion Theory, Addison-Wesley, Redwood City, CA, 1985, p. 406.

5. Mueller, C. J., Driscoll, J. F., Sutkus, D. J., Roberts, W. L., Drake, M. C., and Smooke, M. D., Combust. Flame 100:323-331 (1995).

6. Ratner, A., Driscoll, J. F., Donbar, J. M., Carter, C. D., and Mullin, J. A., Proc. Combust. Inst. 28:245 (2000).

7. Sung, C. J., Liu, J. B., and Law, C. K., Combust. Flame 102:481-494 (1995).

8. Santoro, V. S., Kyritsis, D. C., and Gomez, A., Proc. Combust. Inst. 28:1023-1030 (2000).

9. Santoro, V. S., Kyritsis, D. C., Liñán, A., and Gomez, A., Proc. Combust. Inst. 28:2109-2116 (2000).
10. Lee, J. C., Frouzakis, C. E., and Boulouchos, K., Combust. Sci. Technol. 158:365-388 (2000).

11. Finke, H., and Grünfeld, G., Proc. Combust. Inst. 28:2133-2140 (2000).

12. Roberts, W. L., Driscoll, J. F., Drake, M. C., and Goss, L. P., Combust. Flame 94:58-69 (1993).

13. Santoro, V. S., Liñán, A., and Gomez, A., Proc. Combust. Inst. 28:2039-2046 (2000).

14. Marran, D. F., "Quantitative, Two-Dimensional Laser Diagnostics in Idealized and Practical Combustion Systems," Ph.D. thesis, Department of Mechanical Engineering, Yale University, New Haven, CT 1997.

15. Kyritsis, D. C., Santoro, V. S., and Gomez, A., "Measurements and Computations of Scalar Dissipation Rate in Vortex Perturbed Counterflow Diffusion Flames," paper 72, 2001 ESCI Meeting, Hilton Head, SC, December 3-5, 2001.

16. Frank, J. H., Lyons, K. M., Marran, D. F., and Long, M. B., Proc. Combust. Inst. 25:1159-1166 (1994).

17. Fielding, J., Schaffer, A. M., and Long, M. B., Proc. Combust. Inst. 27:1007-1014 (1998).

18. Stårner, S. H., Bilger, R. W., Lyons, K. M., Frank, J., and Long, M., Combust. Flame 99:347-354 (1994).

19. Coix, P. J., Leonard, K. R., Talbot, L., and Chen, J. Y., Exp. Fluids 15:247-254 (1993).

20. Kelman, J. B., and Masri, A. R., Appl. Opt. 36:35063514 (1997).

21. Stårner, S. H., Bilger, R. W., Dibble, R., and Barlow, R., Combust. Sci. Technol, 86:223-236 (1992).

22. Kyritsis, D. C., Felton, P. G., Huang, Y., and Bracco, F. V., Appl. Opt. 39:6771-6780 (2000).

23. Yoshida, K., and Takagi, T., Proc. Combust. Inst. 27:685-692 (1998).

24. Katta, V. R., Carter, C. D., Fiechtner, G. J., Roquemore, W. M., Gord, J. R., and Rolon, J. C., Proc. Combust. Inst. 27:587-594 (1998).

25. Alden, M., Grafstrom, P., Lundberg, H., and Svandberg, S., Opt. Lett. 8:241-243 (1983).

26. Seitzman, J. M., Kychakoff, G., and Hanson, R. K., Opt. Lett. 10:439-441 (1985).

\section{COMMENTS}

Akio Kitajima, National Institute of Advanced Industrial Science and Technology, Japan. Is it adequate to use the definition of scalar dissipation rate for steady conditions? I am wondering if it is necessary to introduce some unsteady effects to estimate the scalar dissipation rate, such as mixture fraction variations similar to a turbulent case. Do you have any comment on this?
Author's Reply. The definition of the scalar dissipation rate is well established regardless of the steadiness of the flow. Its relationship with the strain rate, that is also commonly used to characterize laminar flames, varies depending on whether conditions are steady or not. Detailed expressions in both cases are provided in Ref. [9] in our paper. 